\title{
THE EFFECT OF A BAR ON THE KINEMATICS OF A CENTRAL RING STRUCTURE IN NGC4736
}

\author{
P. PIŞMIŞ \& E. MORENO \\ Instituto de Astronomia, Apartado Postal 70-264 \\ C.P. 04510, México, D.F.
}

\begin{abstract}
We analyze the kinematics of the gaseous ring in the central region of NGC 4736 considering an ejection mechanism for the formation of the ring in a non-axisymmetric potential. A barred spiral of strength $\mathbf{1 0}$ or 25 per cent of the axisymmetric background is consistent with van der Kruit (1974) observational data.
\end{abstract}

\section{The model}

Extending a recent work (Pişmiş \& Moreno 1992) we have taken a barred spiral galaxy with a composite potential, consisting of an overall axisymmetric potential and a barred one superposed on it, to investigate the motion of material ejected from the nuclear region, taking into account its interaction with the interstellar medium. This interaction leads an ejecta to follow a circular orbit in the axisymmetric case and a deformed circle when a bar plus a two-armed spiral pattern is introduced. This provides a way to form spiral and ring-like structures in galactic inner regions by means of an ejection mechanism. In NGC4736, showing a ring structure, the axisymmetric part of the potential is the one used by Pişmis and Moreno (1992). The non-axysimmetric part is of the form proposed by Roberts et al. (1979), and we have tested a force strength of 10 and 25 per cent of this component with respect to the axisymmetric background. The velocity field of the interstellar gas in the overall potential is taken as that considered by Prendergast (1962), i.e. along the effective equipotential curves in the non-inertial frame defined by the bar-and-spiral pattern.

\section{Results}

In Fig. 1 we show four situations modeling the kinematics of the ring in NGC4736, with $N=1,3$ (see eq. 6 of Roberts et al. 1979) and a bar-spiral strength of 10 and 25 per cent for each value of $\mathrm{N}$. This figure is to be compared with observational data given by van der Kruit (1974, Fig. 3). The ring is built in $\sim 10^{8}$ years, about an order of magnitude greater than in the axisymmetric potential, but the required energy is still of the order $10^{56}-10^{57}$ ergs (Pişmiş \& Moreno 1992). 


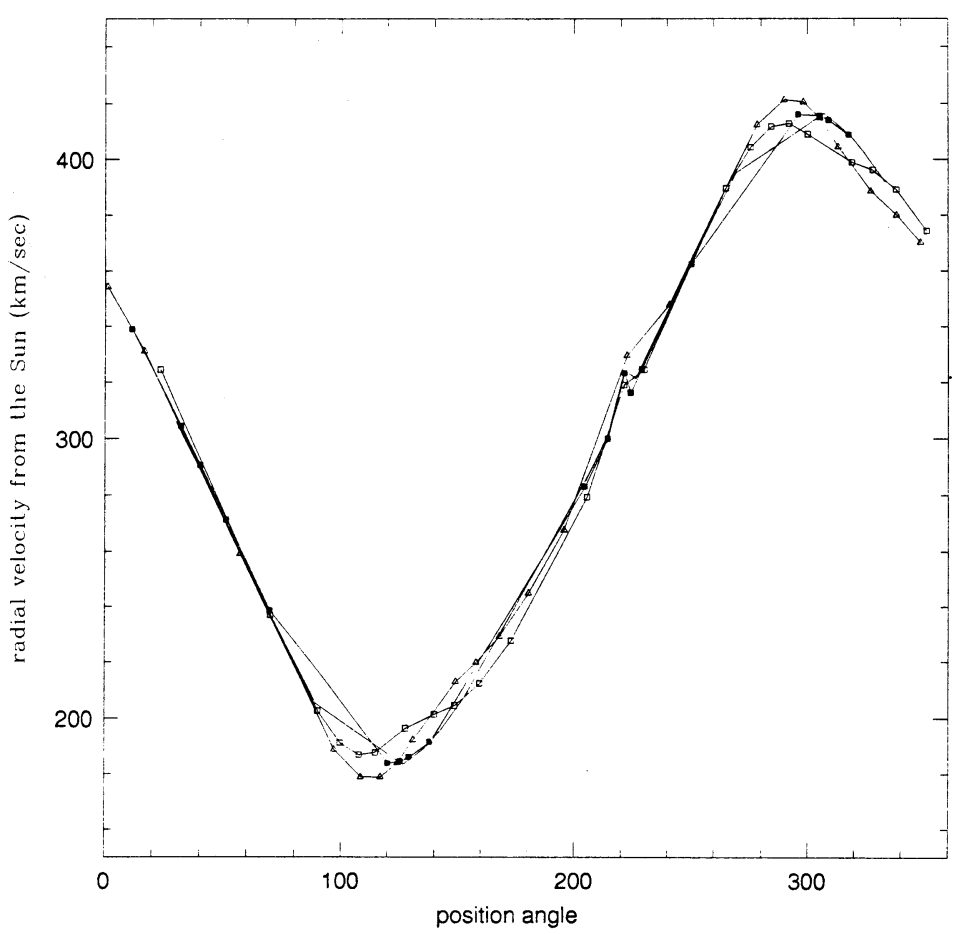

Fig. 1. Kinematics of the ring in NGC 4736 with a bar-and-spiral force strength of 10 per cent (filled symbols) and 25 per cent (open symbols) of the axisymmetric potential.

The large scatter in velocity around position angle 90 and 270 (Fig. 3 of van der Kruit, 1974) can be attached to the interaction of the ejecta with the barred spiral pattern. Sanders \& Bania (1976) gave this interpretation in their single explosion model; here we propose a discrete ejection to form the ring, requiring less power and energy of a central engine.

\section{REFERENCES}

Pişmiş, P. \& Moreno, E. 1992, in preparation.

Prendergast, K.H. 1962, in Interstellar Matter in Galaxies, ed. L. Woltjer, p. 217.

Roberts, W.W., Huntley, J.M., \& van Albada, G.D. 1979, Ap.J., $\underline{\mathbf{2 3 3}}, 67$.

Sanders, R.H. \& Bania, T.M. 1976, Ap.J., $\underline{204}, 341$.

van der Kruit, P.C. 1974, Ap.J., $\underline{188}, 3$. 